\title{
Risk Management Strategies for a Wind Power Producer in Electricity Markets
}

\author{
Mohammadreza Janghorbani*, Seyed Mohammad Shariatmadar, Vahid Amir, \\ Mohammad Ghanbari Jolfaei and Ali Madanimohammadi
}

Department of Electrical Engineering, Naragh Branch, Islamic Azad University, Naragh, Iran; unesbahalke@gmail.com

\begin{abstract}
Under a market framework, the development of optimal offering strategies is crucial for wind power producers to achieve maximum profit. In this paper, a two-stage stochastic programming approach is proposed, considering the uncertainties on wind power production and electricity market prices. An artificial intelligence model allows generating wind-price scenarios. Also, risk management is appropriately addressed. Results from a real-world case study are presented, in order to illustrate the proficiency of the proposed approach.
\end{abstract}

Keywords: Risk, Generation, Wind energy, Uncertainty, Producer

\section{Introduction}

Increasing security and environmental of supply concerns as well as technological improvements compose a favorable environment for the expansion of generators based on Renewable Energy Sources (RES). However, the operation of renewable energy sources such as solar or wind plants presents the inconvenience dependence on the variability of the solar or wind resource. It makes large scale integration of renewable units into power systems specially challenging ${ }^{3,9}$.

Due to the centralized process in several countries, renewable energy generators usually will operate under electricity market conditions. However, electricity markets originally have been designed to integrate power producers having the ability to control their own individual power production at any time, which isn't the case of power producers using non dispatchable RES units. As a result, RES power producers may penalize by electricity market laws ${ }^{1,5}$.

Electricity prices show high variability that reflect dynamic behavior of the market. Furthermore, the power supply that is generated from wind energy is highly alternative. Therefore, decision-makers should be prepared against electricity market prices and also the uncertainties on wind power production ${ }^{2,4,6}$.

Considering the uncertainties on the electricity market prices and wind power production needs stochastic programming. Therefore a two-stage stochastic programming approach is suggested, to divide the set of decisions intrinsic to the problem into two specified stages.

The mentioned uncertainties were handled through time-series models. In this paper, an artificial intelligence model is raised to generate wind-price directions.

Also risk management is incorporated in proposed stochastic programming approach, by restricting the variability of expected profit through the Conditional Value-at-Risk (CVaR) methodology $y^{7,8}$.

The suggested approach allows generating the optimal offers that must be submitted to the day-ahead markets by a wind power producer, due to maximize its expected interest considering a given level of risk.

At the last part of the paper, the experience with the implementation of two-stage stochastic programming approach on a case study, based on a wind farm in Portugal, is reported.

${ }^{*}$ Author for correspondence 


\section{Problem Formulation}

\subsection{Risk Management}

Conditional Value-at-Risk (CVaR) shows an appropriate approach to address the risk management problem of the wind power producer.

$\mathrm{CVaR}$ is the expected profit not exceeding a measure $\zeta$ that is called Value-at-Risk (VaR) as:

$$
\mathrm{CVaR}=\mathrm{E}(\mathrm{B} \mid \mathrm{B} \leq \zeta)
$$

Value-at-Risk (VaR) is a measure calculated as the maximum profit value so that the possibility of the profit being equal or lower than this value is equal or lower than $1-\alpha$. It is indicated in the following constraint:

$$
\operatorname{VaR}=\max \{\mathrm{x} \mid \mathrm{p}(\mathrm{B} \leq \mathrm{x}) \leq 1-\alpha\}
$$

Usually the value of $\alpha$ is set between 0.90 and 0.99 . In this paper, $\alpha$ is assumed equal to 0.95 .

CVaR mathematically can be shown as equation 5 if:

$$
-\mathrm{B}_{\mathrm{s}}+\zeta-\eta_{\mathrm{s}} \leq 0
$$

and

$$
\begin{gathered}
\eta_{s} \geq 0 \\
\max \zeta-\frac{1}{1-\alpha} \sum_{s=1}^{s} \rho_{s} \eta_{s}
\end{gathered}
$$

In constraint (3), $\eta_{s}$ is a variable that is zero if the scenario $s$ has a profit bigger than $\zeta$. $\eta$ s for remaining scenarios, is the difference of corresponding profit and $\zeta$. The concept of CVaR is shown in Figure 1.

For stochastic problems VaR has more complexity, and also it is needed to use binary variables for modeling.

But CVaR calculation can be modeled by the simple use of linear constraints and it doesn't need to use binary variables.

\subsection{Objective Function}

A profit maximization risk constrained decision making problem for a wind power producer within the market framework can be summarized in equation (6). This Formula to be maximized contains the operational costs, the CVaR of profit, and expected profit. In this equation,

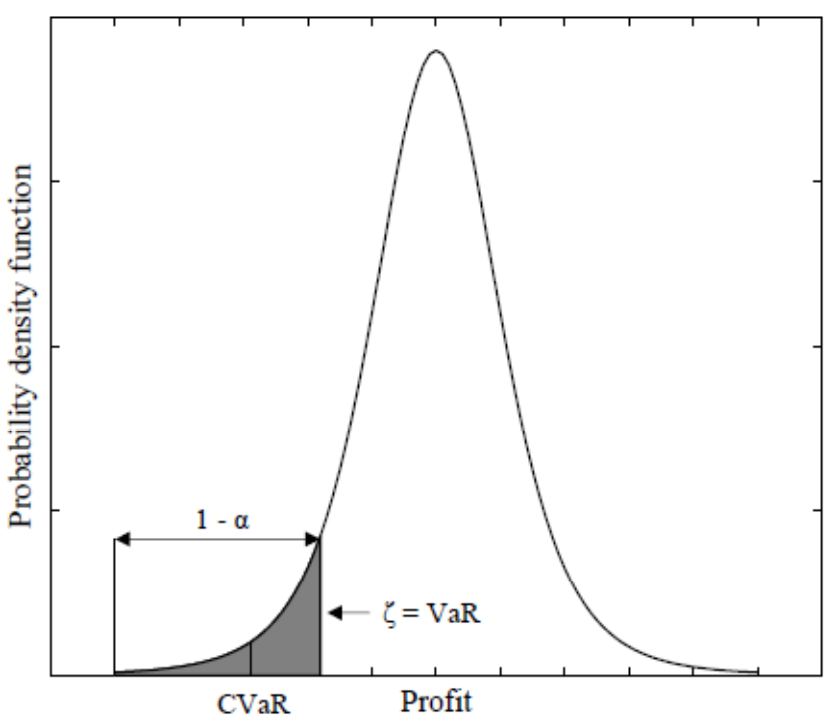

Figure 1. VaR and CVaR illustration ${ }^{3}$.

$\mathrm{S}$ represents set of scenarios and $\mathrm{H}$ shows set of hours and $\mathrm{I}$ is the number of wind turbine.

$$
F=\sum_{s=1}^{s} \rho_{s} \sum_{h=1}^{H}\left[\left(p_{s h} \lambda_{s h}\right)-P d e v_{s h}\right]-\sum_{h=1}^{H} \sum_{i=1}^{I}\left[(g)_{i n} b_{i n}\right]
$$

$$
+\beta\left(\zeta-\frac{1}{1-\alpha} \sum_{s=1}^{s} \rho_{s} \eta_{s}\right)
$$

$\mathrm{p}_{\mathrm{sh}}$ is the wind farm's power output in duration $\mathrm{h}$ in that scenario. $\lambda_{s h}$ indicates forecasted price in duration $\mathrm{h}$ in related scenario while $\rho_{s}$ is equal to possibility of happening of scenario and Also Pdev ${ }_{s h}$ represents penalization for the wind farm's deviation in duration $h$. In the next part of equation $b_{i h}$ ndicates operational cost at duration $\mathrm{h}$ associated to wind turbine $\mathrm{i}$ and $g_{\text {ih }}$ shows output of power for the wind turbine $i$ in duration $h$ and $\beta$ indicates weighting factor to achieve proper tradeoff between risk and profit ${ }^{5}$.

The interest is given by the product of the expected electricity market price by the output of power in the wind farm has the following equation:

$$
\mathrm{L}_{\mathrm{sh}}=\mathrm{p}_{\mathrm{sh}} \lambda_{\mathrm{sh}}
$$

Here, the deviations are calculated in absolute value, and they come from deficit or excess of energy as illustrated in the following equation:

$$
\operatorname{dev}_{\mathrm{sh}}=\left|\mathrm{x}_{\mathrm{h}}-\mathrm{p}_{\mathrm{sh}}\right|
$$


For producers, the penalty for deviation is corresponding to the product of cost for the shifted power in absolute value as comes in the next relation:

$$
P_{d e v_{s h}}= \begin{cases}\lambda_{s h} \operatorname{deg}_{s h} r_{s h}^{+}, & d e v_{s h} \geq 0 \\ \lambda_{s h} \operatorname{dev} v_{s h} r_{s h}^{-}, & \text {dev } v_{s h}<0\end{cases}
$$

It is important to note that the expected profit is the difference between operational costs, revenue of the wind farm and penalty for deviation.

By substituting equation (9) into equation (6) we have:

$$
\begin{aligned}
F= & \sum_{s=1}^{s} \rho_{s} \sum_{h=1}^{H}\left[\lambda_{s h} P_{s h}-\lambda_{s h} r_{s h}^{-} d_{s h}^{-}-\lambda_{s h} r_{s h}^{+} d_{s h}^{+}\right] \\
& -\sum_{s=1}^{s} \sum_{i=1}^{I} b_{i h} g_{i h}+\beta\left(\zeta-\frac{1}{1-\alpha} \sum_{s=1}^{s} \eta_{s} \rho_{s}\right)
\end{aligned}
$$

\subsection{Modalities}

For a total offset in the relation $\operatorname{dev}_{\mathrm{sh}}=\mathrm{d}_{\mathrm{sh}}^{+}-\mathrm{d}_{\mathrm{sh}}^{-}$, the solution is guaranteed to be achieved with one of these variables $\mathrm{d}_{\mathrm{sh}}^{-}$or $\mathrm{d}_{\mathrm{sh}}^{+}$equal to zero, because $\mathrm{d}_{\mathrm{sh}}^{-} \geq 1$ and $\mathrm{d}_{\mathrm{sh}}^{+} \leq 1$ :

$$
\mathrm{p}_{\mathrm{sh}}-\mathrm{x}_{\mathrm{h}}+\mathrm{d}_{\mathrm{sh}}^{-}-\mathrm{d}_{\mathrm{sh}}^{+}=0
$$

To make offers for the market, it is needed to satisfy the technical limitations of the wind farm. Therefore, the optimal value of objective function is determined if inequality constraints or simple bounds on variables.

These constraints are shown below:

$$
\begin{aligned}
& 0 \leq d_{s h}^{-} \leq P_{\max } \\
& 0 \leq d_{s h}^{+} \leq W_{s h}
\end{aligned}
$$

In above constraints, $P_{\max }$ is maximum power installed in the wind farm and $\mathrm{W}_{\mathrm{sh}}$ is forecasted wind power production in period $h$ in scenario s. Constraints (12) and (13) force caps on the negative and positive deviations.

Constraint (14) shows that offers must be equal or lower than the total power output of the wind turbines and indicated as follows:

$$
x_{h} \leq \sum_{i=1}^{I} g_{i h}
$$

In Constraint (15), the offers are restricted by the maximum power installed on the wind farm and we have:

$$
0 \leq \mathrm{x}_{\mathrm{h}} \leq P_{\max }
$$

$\eta_{s}$ in equation (17) is a variable equal to zero if the scenario $s$ has a profit bigger than $\zeta$. In the other scenarios, $\eta_{s}$ is equal to the difference of the corresponding profit and $\zeta$ :

$$
\begin{gathered}
\eta_{s} \geq 0 \\
-\sum_{h=1}^{H}\left[\lambda_{s h} P_{s h}-\lambda_{s h} r_{s h}^{+} d_{s h}^{+}-\lambda_{s h} r_{s h}^{-} d_{s h}^{-}-\sum_{i=1}^{I} b_{i h} g_{i h}\right] \\
+\zeta-\eta_{s} \leq 0
\end{gathered}
$$

\subsection{The Objective Function Linearization}

The function given in the previous section, is characterized by nonlinearities because of an absolute value. Therefore, it is needed to use one mathematical process that lets us reformulating into linear problem.

In this section, the problem which has involvement with absolute value terms is transformed into one standard linear programming formulation. At first, it is considered that if:

$$
\begin{aligned}
X^{\min } & \leq X \leq X^{\max } \\
& x \in R^{n}
\end{aligned}
$$

Then we have the equation (20). In this equation, c represents vector of coefficients for linear term and the function $F($.$) is the objective function of decision fac-$ tors or variables. The variable $\mathrm{x}$ is one set of decisions variables.

$$
\operatorname{Max} F=C^{T} x-|x|
$$

In constraint (18), $X^{\max }$ and $X^{\min }$ are the upper and lower restriction vectors on variables.

Then, absolute valued variables are changed with two variables that are strictly positive as indicated in the following equation:

$$
|x|=X^{-}+X^{+}
$$

Moreover, variables are substituted by the difference of the same two positive variables:

$$
X=X^{+}-X^{-}
$$

Now, the equivalent linear programming problem is computed by the the equation (26) if: 


$$
\begin{gathered}
X^{\min } \leq X \leq X^{\max } \\
X=X^{+}-X^{-} \\
X^{+} \geq 0, X^{-} \geq 0 \\
\operatorname{Max} F=C^{T} x-\left(\mathrm{x}^{+}+\mathrm{x}^{-}\right)
\end{gathered}
$$

\section{Proposed Approach}

\subsection{Scenario Tree}

Figure 2 illustrates a scenario tree which is used to represent the first and second-stage decisions.

Because of tractability for the problem it is better to reduce the volume or size of the scenario tree. One technique for scenario-reduction provides an efficient way to select an instance subset of scenarios which covers most scenario realizations.

\subsection{Two-stage Stochastic Programming}

This two-stage stochastic programming approach can formulate as below:

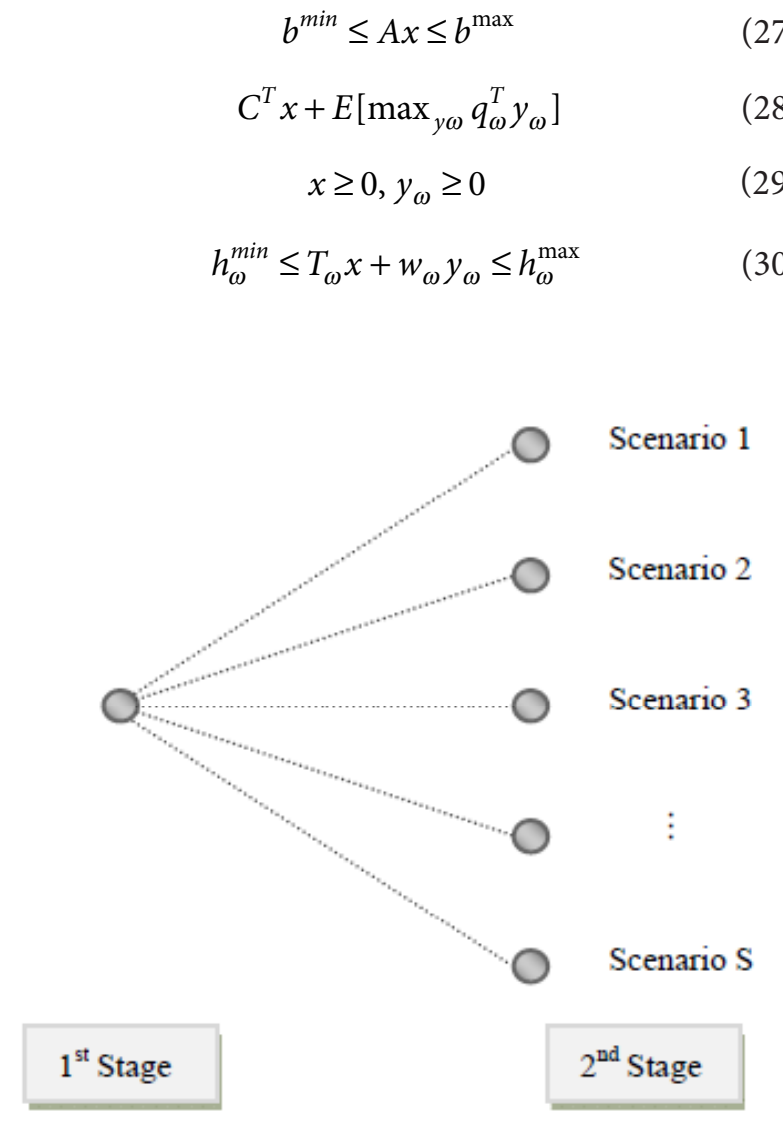

Figure 2. Scenario tree ${ }^{5}$.
In these equations $\mathrm{b}_{\max }$ and $\mathrm{b}_{\text {min }}$ are the upper and lower boundary vectors for the constraints of first-stage, $A$ is the coefficients matrix for the first-stage constraints while $\mathrm{c}$ is the objective function coefficients vector in the first-stage for x variables. $T_{\omega}$ is the technology matrix for each $\omega, H_{\omega}^{\max }$ and $H_{\omega}^{\min }$ are the upper and lower boundary vectors for the constraints of second-stage and under scenario $\omega, W_{\omega}$ is the recourse matrix and $q_{\omega}$ indicates the coefficients vector for the variables of second-stage in the linear term.

At the first stage, before the uncertainties indicated by $\mathrm{x}$ are known, the decision must be taken. The first stage decision of $\mathrm{x}$ just depends on the information available until that time. At the second stage, the decision is made about the vector $y$, while the information $x$ is already available.

Two stages problem means that the decision $\mathrm{x}$ is independent of the results of the second-stage, and therefore the vector $\mathrm{x}$ is equal for all events that are possible and may happen in the second stage of the problem ${ }^{5}$.

\subsection{Problem of Deterministic Equivalent}

Usually the stochastic model is a complex computational problem, therefore it is usual to select the deterministic model solution by use of the average of several random variables or solving a deterministic problem for each scenario ${ }^{5}$. Previously shown problem is equivalent to the so-called deterministic equivalent one that is shown in equation (34) if we have:

$$
\begin{gathered}
x \geq 0, y_{s} \geq 0 \quad \text { For } s=1, \ldots, \mathrm{S} \\
b^{\min } \leq A x \leq b^{\max } \\
h_{s}^{\min } \leq T_{s} x+w_{s} y_{s} \leq h_{s}^{\max } \text { For } \mathrm{s}=1, \ldots, \mathrm{S} \\
\operatorname{Max}_{x, y_{s}} C^{T} x+\sum_{s=1}^{s} \rho_{s} q_{s}^{T} y_{s}
\end{gathered}
$$

\section{Case Study}

Previously discussed stochastic programming approach has been applied on a case study, a wind farm located in the Viana do Castelo region in Portugal. Total installed wind power capacity is 66 Mega Watts, corresponding to 33 2.0-Mega Watts wind turbines. This model has been implemented in MATLAB software and solved using the optimization solver package (CPLEX). Numerical testing has been performed on a system with 2 GB RAM and 2- $\mathrm{GHz}$ processor. 


\subsection{Input Data}

The suggested approach talks about the uncertainty in both electricity market prices and wind power production using the scenarios in a stochastic problem. The profits of a wind power producer are weighing with regard to a given risk level. Based on the rules, imbalance penalties are imposed to secure better system operation and to prevent gaming.

The time horizon used in this case is one day divided into 24 hourly periods. This case study is consists of ten wind power production scenarios as shown in Figure 3, and ten electricity market prices scenarios as shown in Figure 4. Although the number of scenarios selected arbitrarily, describes the stochastic processes adequately. Moreover, ten scenarios for imbalance price ratio are taken

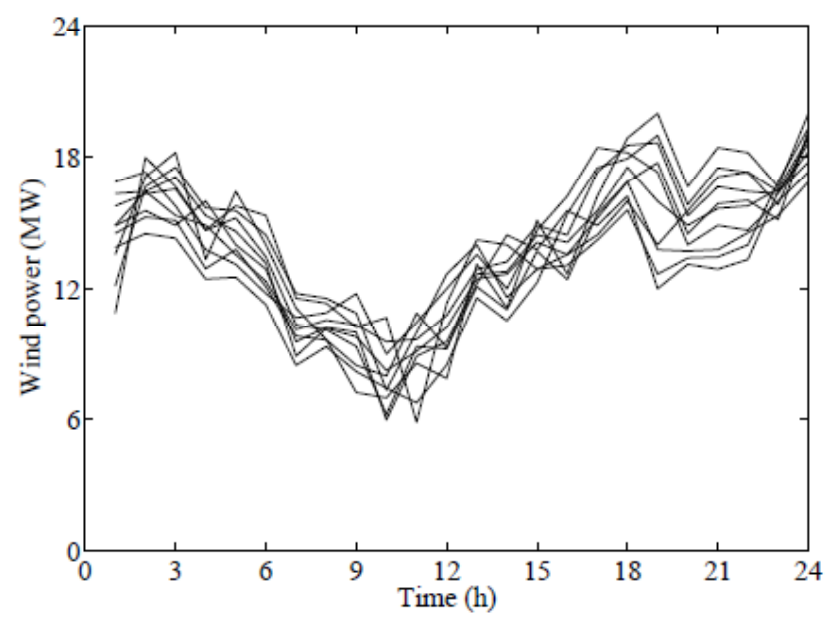

Figure 3. BWind power production scenarios ${ }^{5}$.

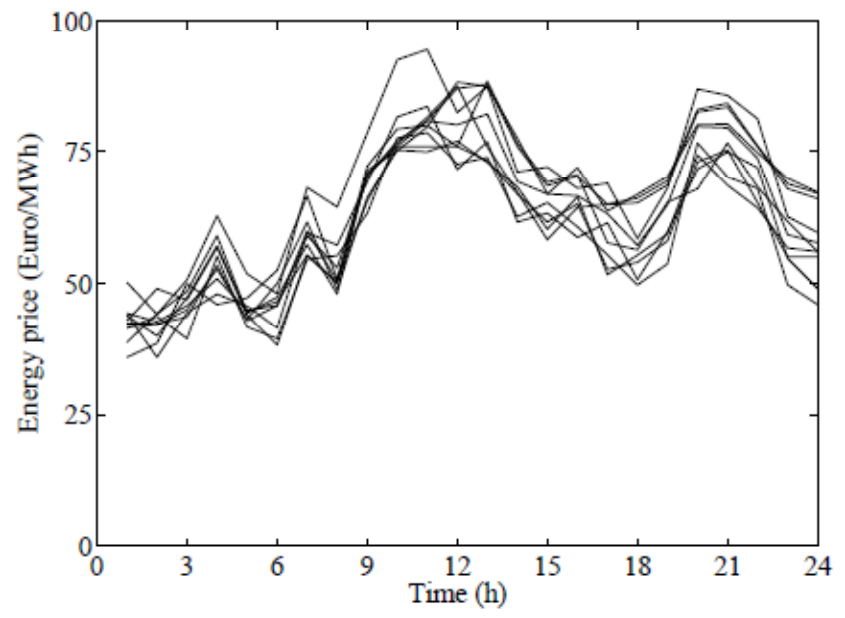

Figure 4. Electricity market price scenarios ${ }^{5}$. into account. Therefore, the overall number of scenarios that is generated in this optimization problem is $S=1000$. For each generated scenario the probability is $1 / \mathrm{S}$.

\subsection{Results Analysis}

A complete comparison of the offering strategies in the market for various risk levels using the suggested approach is presented thereafter.

The solution of the optimization model for the daily market content of the optimal bids. The optimal bids are common to the 1000 scenarios (Figure 5).

The two-stage stochastic programming consists of $(\mathrm{S}+1+\mathrm{H} .(3 \times \mathrm{S}+\mathrm{I}+1))$ variables which are continuous and $(S+H .(S+1))$ constraints. So the size of the problem depends on the number of scenarios.

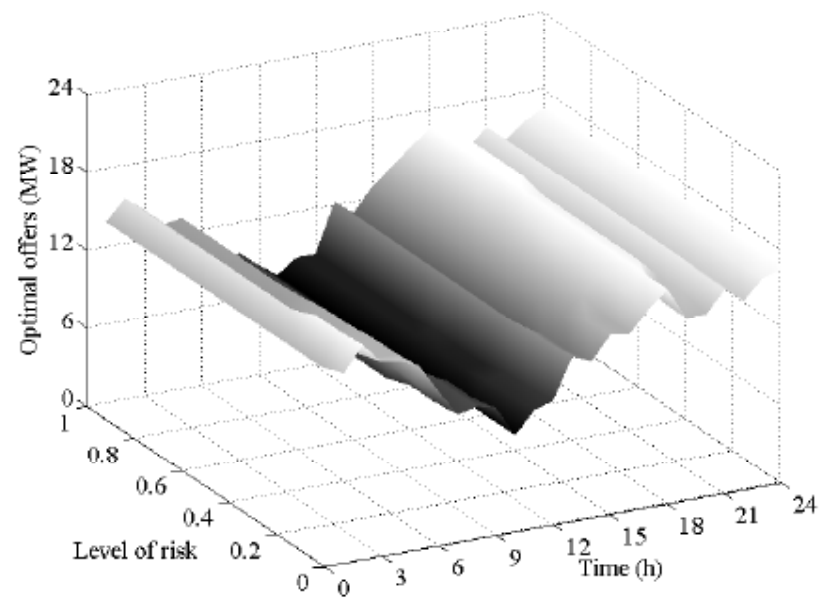

Figure 5. Optimal hourly bids for different risk levels ${ }^{5}$.

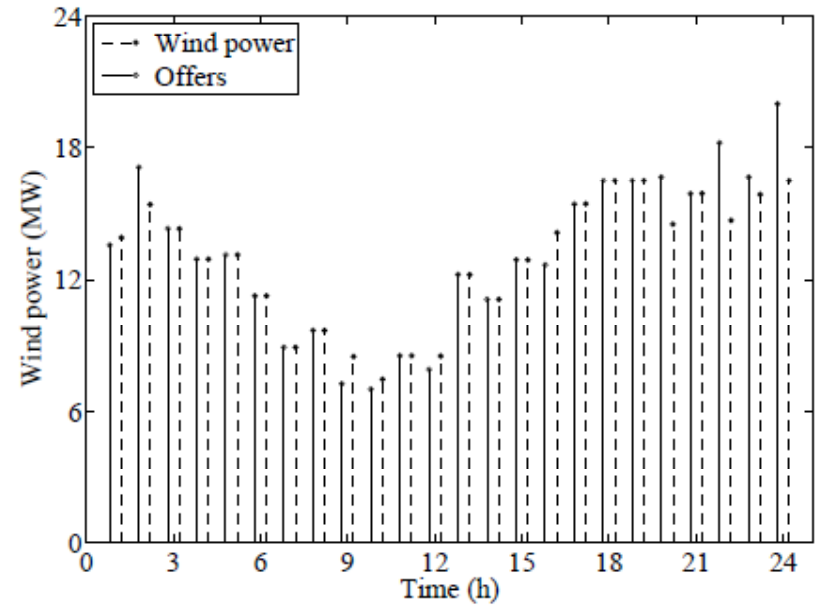

Figure 6. Wind power production, for risk level $\beta=1$ and optimal offers to be submitted to the future market ${ }^{5}$. 
Figure 5 illustrates the ability of a wind power producer to commerce in the day-ahead market with regard to the desired risk level.

To choose one scenario of the problem, it can be seen in Figure 6 that the wind farm regulates its production to minimize deviations. Nonetheless, in about every hour there are small variances between the power output of the wind farm and the offers. The deviations that result from the difference between the wind power production and the offers are illustrated in Figure 7. The positive deviation means that the production of wind power was higher than the offer submitted to the market, and vice-versa.

Profit standard deviation versus the expected profit is shown in Figure 8, it has seven values for $\beta$ and $\alpha=0.95$ in all examples.

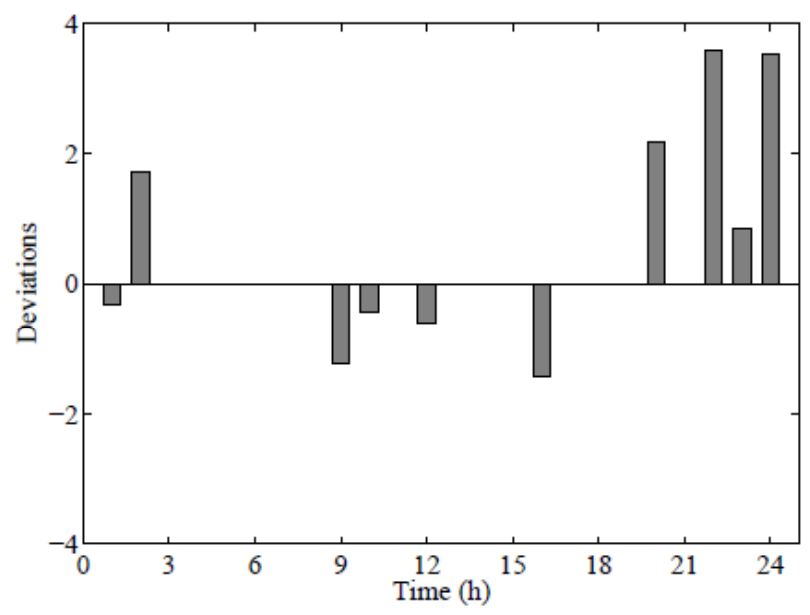

Figure 7. Deviations from difference between the wind power production for a risk level $\beta=1$ and the offers ${ }^{5}$.

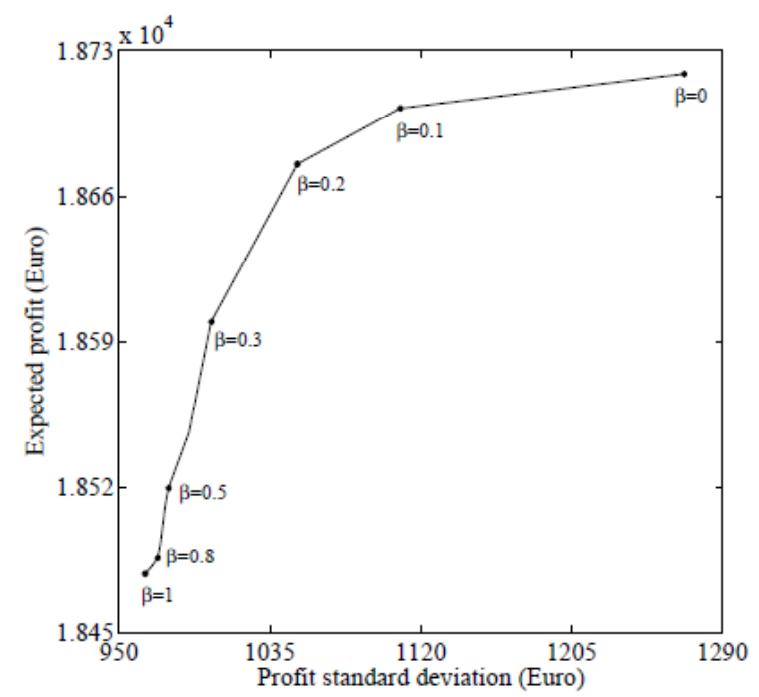

Figure 8. Expected profit versus profit standard deviation ${ }^{5}$.
Figure 8 provides the maximum expected profit that is achievable for each risk level or, alternatively, the minimum achievable risk level for each expected profit. Such figure known as efficient frontier, reveals that for a risk-neutral producer $(\beta=0)$ expected profit is $18719 €$ with $1268 €$

Table 1. Comparison of the increase in profit for several risk levels

\begin{tabular}{lcccc}
\hline $\begin{array}{l}\text { Risk } \\
\text { Level }\end{array}$ & $\begin{array}{c}\text { Profit Std. } \\
\text { Deviation }(€)\end{array}$ & $\begin{array}{c}\text { Expected } \\
\text { Profit (€) }\end{array}$ & \% Increase & $\begin{array}{c}\text { CPU } \\
\text { Time (s) }\end{array}$ \\
\hline 1.0 & 965 & 18478 & - & 1.62 \\
0.8 & 971 & 18486 & 0.04 & 1.05 \\
0.5 & 978 & 18519 & 0.22 & 0.98 \\
0.3 & 1001 & 18599 & 0.65 & 0.92 \\
0.2 & 1050 & 18675 & 1.07 & 0.88 \\
0.1 & 1108 & 18702 & 1.21 & 0.82 \\
0.0 & 1268 & 18719 & 1.30 & 0.76 \\
\hline
\end{tabular}

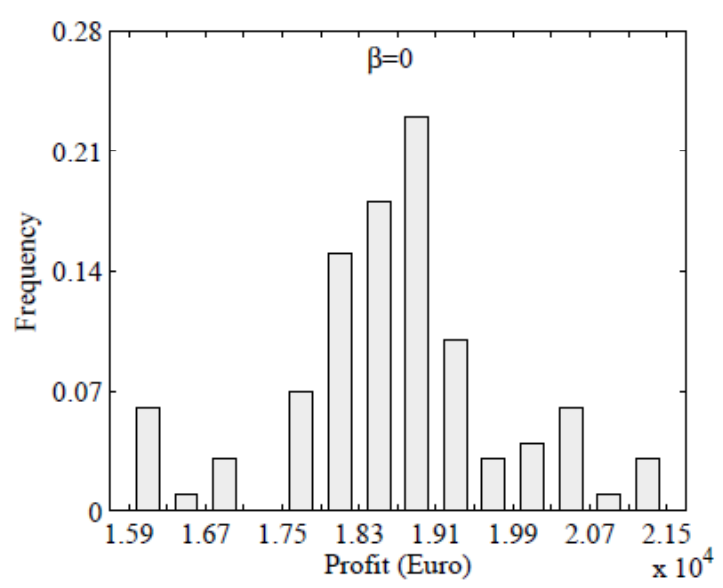

Figure 9. Histogram of the profits for the risk level corresponding to $\beta=0$ (Catalão et al., 2011).

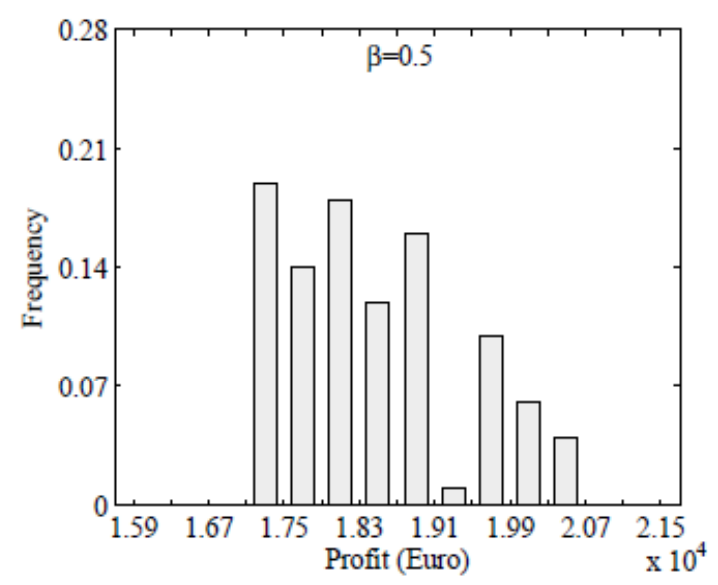

Figure 10. Histogram of the profits for the risk level corresponding to $\beta=0.5$ (Catalão et al., 2011). 
standard deviation. While a risk-averse producer $(\beta=1)$ expects to reach to a profit of $18478 €$ with a lower standard deviation of $965 €$. A numerical comparison of the increase in profit for several risk levels illustrated in Table 1.

The maximum profit represents an increase of $1.30 \%$ with regard to risk level $\beta=0$. Nevertheless, the profit standard deviation is higher for $\beta=0$.

Figures 9 and 10 illustrate the histograms of the profits for $\beta=0$ and $\beta=0.5$, respectively.

Analyzing Figures 9 and 10, it can be verified that the risk level corresponding to $\beta=0$ implies a higher expected profit than for $\beta=0.5$. Nevertheless, $\beta=0$ is riskier than $\beta=0.5$ because financial loss can happen under some scenarios, thus a risk-averse producer may prefer $\beta=0.5$. Therefore, our approach allows selecting best solution with regard to the level of desired risk.

\section{Conclusion}

In this paper, a two-stage stochastic programming approach is proposed to improve risk-constrained offering strategies for a wind power producer. A hybrid intelligent approach generates wind-price scenarios, and risk management is also incorporated by restricting the volatility of expected profit through the CVaR methodology. Also a thorough comparison of optimal offering strategies in the market for various risk levels is presented in this paper. Furthermore, the presented results on a realistic case study validate proficiency of the proposed approach, enabling the selection of the best solution with regard to the desired risk exposure level, while the average calculation time is acceptable.

\section{References}

1. Boqiang R, Chuanwen J. A review on the economic dispatch and risk management considering wind power in the power market. Renew Sustain Energ Rev. 2009; 13:2169-74.

2. Botterud A, Wang J, Bessa R, Keko H, Miranda V. Risk management and optimal bidding for a wind power producer. Power and Energy Society General Meeting; 2010; IEEE. p. 1-8.

3. Botterud A, Zhou Z, Wang J, Bessa RJ, Keko H, Sumaili J, Miranda V. Wind power trading under uncertainty in LMP markets. IEEE Trans Power Syst. 2012; 27:894-903.

4. Bourry F, Juban J, Costa L, Kariniotakis G. Advanced strategies for wind power trading in short-term electricity markets. Proceedings European Wind Energy Conference \& Exhibition EWEC; 2008.

5. Catalão J, Pousinho H, Mendes V. Development of riskconstrained offering strategies for a wind power producer under uncertainty. Constraints. 2011; 1, 10.

6. Pinson P, Chevallier C, Kariniotakis GN. Trading wind generation from short-term probabilistic forecasts of wind power. IEEE Transactions on Power Systems. 2007; 22:1148-56.

7. Galloway S, Bell G, Mcdonald J, Burt G, Siewierski T. Managing the risk of trading wind energy in a competitive market. IEE Proc Generat Transm Distrib. 2006; 106-14.

8. Hiroux C, Saguan M. Large-scale wind power in European electricity markets: Time for revisiting support schemes and market designs? Energy Policy. 2010; 38:3135-45.

9. Li X, Jiang C. Short-term operation model and risk management for wind power penetrated system in electricity market. IEEE Trans Power Syst. 2011; 26:932-39. 